\title{
An algorithm to improve lateralization accuracy of inferior petrosal sinus sampling: procedural nuances for complex patterns of venous drainage. Patient series
}

\author{
Abhiijth V. Matur, MD, ${ }^{1}$ Alaina M. Body, MD, ${ }^{2}$ Mark D. Johnson, MD, ${ }^{2}$ Matthew S. Smith, MD, ${ }^{3}$ Ruchi Bhabhra, MD, PhD, ${ }^{4}$ \\ Emily J. Lester, RN, ASN, ${ }^{5}$ Trisha L. Stahl, AAS, ${ }^{5}$ Aaron W. Grossman, MD, PhD, ${ }^{3}$ Peyman Shirani, MD, ${ }^{3}$ Jonathan A. Forbes, MD, ${ }^{2}$ \\ and Charles J. Prestigiacomo, MD²

\begin{abstract}
${ }^{1}$ Department of General Surgery, University of Kentucky College of Medicine, Lexington, Kentucky; and Departments of ${ }^{2}$ Neurosurgery, ${ }^{3}$ Neurology, ${ }^{4}$ Endocrinology, and
${ }^{5}$ Radiology, University of Cincinnati College of Medicine, Cincinnati, Ohio
\end{abstract}

\begin{abstract}
BACKGROUND Inferior petrosal sinus sampling (IPSS) is a useful technique in the diagnosis of Cushing's disease (CD) when the imaging finding is negative or equivocal. Different authors have reported considerable variability in the ability to determine tumor laterality with IPSS. Here the authors present a retrospective case series of 7 patients who underwent IPSS using a systematic algorithm to improve lateralization accuracy by identifying optimal sampling sites on the basis of individual cavernous sinus drainage patterns in each patient.

OBSERVATIONS Of the 7 patients identified, 6 were determined to have CD and subsequently underwent surgery. IPSS was accurate in all patients from whom laterality was predicted. Arterial and venous angiography were used to define cavernous sinus drainage patterns and determine optimal sampling sites. All patients who underwent surgery achieved hormonal cure.

LESSONS All IPSS predictions of lateralization were correct when available, and all patients who underwent surgery achieved hormonal cure. Advances in angiographic techniques for identification of the site of primary drainage from the cavernous sinus and subsequent optimization of microcatheter placement may improve the ability to predict tumor laterality.
\end{abstract}

https://thejns.org/doi/abs/10.3171/CASE21374

KEYWORDS Cushing's disease; pituitary; inferior petrosal sinus sampling; IPSS; endovascular

Bilateral inferior petrosal sinus sampling (IPSS) is a technique that is used to diagnose Cushing's disease, especially when no evidence of pituitary adenoma is present on magnetic resonance imaging (MRI) in the setting of adrenocorticotropic hormone (ACTH)dependent Cushing's syndrome. ${ }^{1,2}$ The technique takes advantage of the short half-life of ACTH, which results in a concentration gradient between the inferior petrosal sinus (IPS) and peripheral blood. $^{1,2}$ IPSS is performed bilaterally and involves simultaneous blood draws at regular intervals after stimulation with corticotropinreleasing hormone $(\mathrm{CRH})$. A central/peripheral $\mathrm{ACTH}$ ratio $>3$ after $\mathrm{CRH}$ administration has a sensitivity of $81 \%-100 \%$ and a specificity of $90 \%-95 \%$ for the diagnosis of Cushing's disease. ${ }^{3,4}$ An intersinus ACTH gradient $>1.4$ is typically used to determine laterality and has a positive predictive value that is generally believed to be between $48 \%$ and $78 \%{ }^{2,5,6}$

Although IPSS is a useful technique in the diagnosis of Cushing's disease, lateralization of microadenomas using IPSS remains challenging. ${ }^{2,5,7,8}$ Technical failure of lateralization in IPSS is often due to anomalous venous drainage, which can confound lateralization by asymmetrically directing the flow of ACTH-carrying venous blood. ${ }^{9}$ Some groups have proposed normalizing venous outflow on the basis of prolactin levels, with promising results. ${ }^{10-14}$ However, there have been no systematized algorithms reported in the literature for overcoming the confounding effects of asymmetrical or anomalous venous drainage. Although there has been one report of successful external jugular vein ACTH sampling in a case of a

ABBREVIATIONS ACTH = adrenocorticotropic hormone; $C D=$ Cushing's disease; $C R H=$ corticotropin-releasing hormone; IPS = inferior petrosal sinus; IPSS = inferior petrosal sinus sampling; MRI = magnetic resonance imaging.

INCLUDE WHEN CITING Published September 27, 2021; DOI: 10.3171/CASE21374.

SUBMITTED June 24, 2021. ACCEPTED July 19, 2021.

(c) 2021 The authors, CC BY-NC-ND 4.0 (http://creativecommons.org/licenses/by-nc-nd/4.0/). 


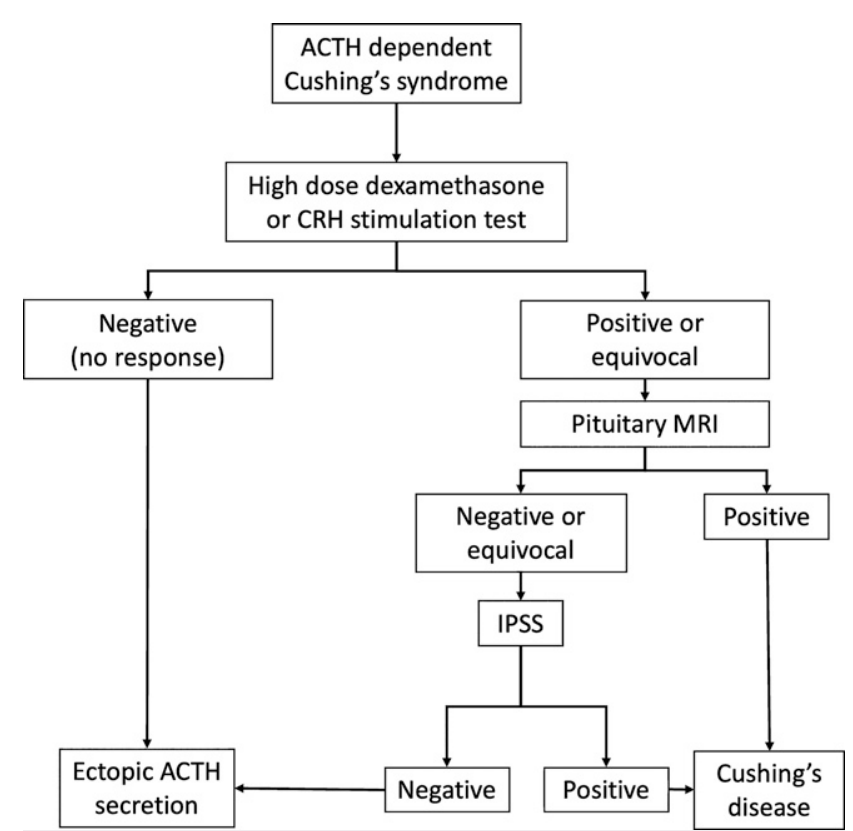

FIG. 1. Algorithm for the diagnosis of Cushing's disease from an ACTH-dependent Cushing's syndrome. IPSS is used when pituitary $\mathrm{MRI}$ is unable to identify a likely ACTH-secreting adenoma.

hypoplastic IPS, there is no systematic approach available to address these cases. ${ }^{15}$ Here we report the systematic approach and algorithm to IPSS used at the University of Cincinnati Medical Center, which combines careful identification of optimal sampling sites with previously described ACTH normalization methods. ${ }^{11,13,14}$ Our addition to commonly used techniques is the careful identification of the site of primary drainage to determine optimal sampling sites in a systematic manner, which has not been previously described. We begin with venography to determine the anatomy of the IPS and escalate to an arterial angiogram with delayed roadmapping if necessary to delineate venous drainage. Optimal sampling sites are then selected on the basis of individual drainage patterns that are based on the site of primary drainage from the cavernous sinus. We believe these methods result in improved lateralization accuracy, thus minimizing unnecessary pituitary exploration and facilitating hormonal cure.

\section{Study Description}

\section{Methods and Endovascular Technique}

In this retrospective case series, we evaluated whether laterality indicated by IPSS using our algorithm correlated with tumor laterality as reported intraoperatively. Patient charts and angiographic images were reviewed in accordance with an institutional review board protocol. IPSS in all patients was performed by the same senior author (C.J.P.). Patients with ACTH-dependent hypercortisolism were referred for IPSS on the basis of the algorithm depicted in Fig. 1. All patients who underwent surgery after diagnosis of Cushing's disease were operated on via a transsphenoidal approach by the same senior author (J.A.F.).

IPSS is a human resources-intensive procedure to ensure safe and accurate placement of catheters along with appropriate processing of specimens. The patient is properly sedated once brought

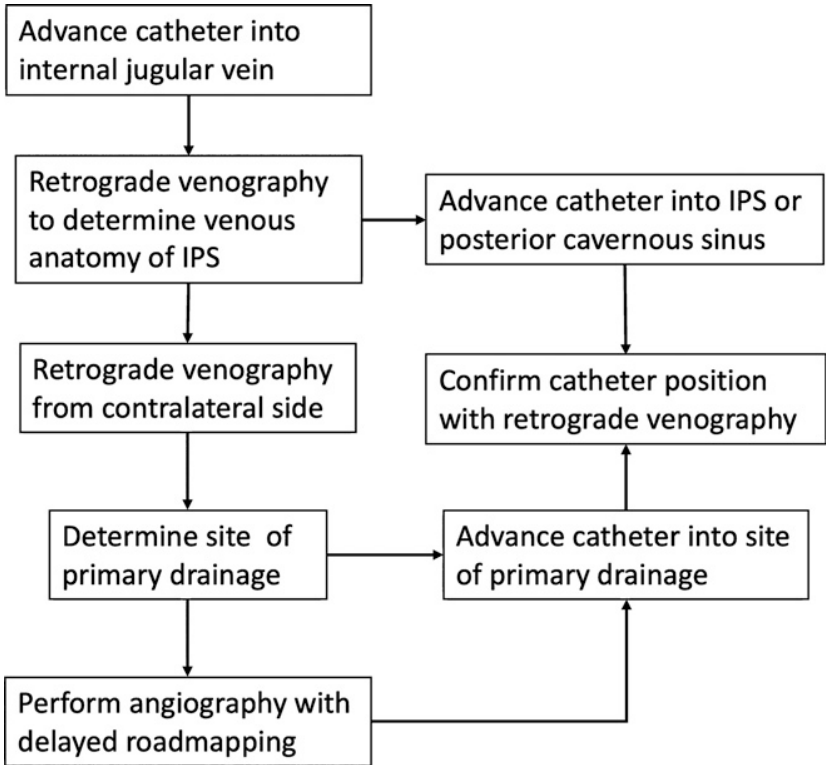

FIG. 2. IPSS procedural algorithm used in each of the patients. We attribute this systematic approach to the improved accuracy of lateralization in our series. In the event of an IPS that cannot be cannulated or visualized, we perform retrograde venography to determine the site of primary drainage from the cavernous sinus. If this fails to identify an optimal sampling location, we perform contralateral venography and finally arterial angiography with delayed roadmapping.

to the angiography suite, where both groins are shaved, prepped, and draped in a sterile fashion. Using the modified Seldinger technique, the right common femoral vein is accessed percutaneously with the patient under local anesthetic, and a 5-French sheath is inserted over a wire and secured in place. After angiographic confirmation of position, a heparinized saline flush $(1,000 \mathrm{U} / \mathrm{L})$ is infused. In like fashion, a 6-French sheath is inserted into the left common femoral vein after percutaneous access. The larger sheath size on the left allows reliable peripheral sampling throughout the procedure without the need for peripheral sampling from smaller-caliber upper extremity lines. Of note, the 3-way stopcock on the left sheath is isolated from the remainder of the sterile field. After administering a 1,000-U heparin intravenous bolus, 5-French Envoy catheters (Codman Neuro) are advanced over a Glidewire (Terumo Medical Corp.) into the inferior vena cava and subsequently the internal jugular veins under subtracted fluoroscopic guidance.

The following steps are shown in the algorithm in Fig. 2. We believe that this algorithm provides a systematic approach to cannulating and successfully sampling ACTH-rich venous blood from the site of primary drainage of the cavernous sinus. A retrograde venogram performed at the jugular bulb is used to identify the IPS before advancing a Marksman microcatheter (Medtronic) with a Synchro 0.014-in. microwire (Stryker Neurovascular). Venography is used to confirm the IPS as the site of primary drainage. However, normal variation in IPS anatomy can result in a small or anomalous IPS that is not the site of primary drainage and may be difficult to visualize and cannulate. In this case, we perform retrograde venography from the contralateral side to visualize the site of primary drainage ipsilaterally. If still no drainage pattern is visualized on the ipsilateral or contralateral retrograde venograms, arterial angiography via a 5-French 
selective catheter through a right-sided 5-French sheath can be performed with delayed roadmapping to identify the primary venous drainage pattern of the cavernous sinus. A 5-French arterial sheath is preferred on the right side to reduce the chance of fistulous complications by placing a sheath on the left side (where a larger 6-French venous sheath is located) and to reduce infection risk because the left represents the site of peripheral sampling. After placing the catheter into the IPS, cavernous sinus, or posterior venous plexus, venography is used once more to confirm the position of the catheter. Ideal catheter placement is in the anterior IPS or cavernous sinus, depending on the venous drainage pattern. ${ }^{16}$ If the patient had significant anterior drainage that could not be sampled posteriorly, sampling would be done through the external jugular vein or pterygoid plexus. In the case of a venous plexus instead of an IPS, the catheter would be advanced into the plexus or into the juncture where it meets the internal jugular vein.

With bilateral sites of primary drainage catheterized, blood is obtained from the peripheral site as well as the site of primary drainage bilaterally at -5 minutes, 0 minutes (time of $1 \mu \mathrm{g} / \mathrm{kg} \mathrm{CRH}$ injection), +2 minutes, +5 minutes, and +10 minutes for a total of $6 \mathrm{~mL}$ in each specimen, which is divided in 2 aliquots. All central and peripheral sampling is done simultaneously by 3 teams dedicated to peripheral, right IPS, and left IPS sampling. To avoid confusion, each dedicated team member wears a color-coded sticker on their scrub hat that is matched to a colored sticker on the vial of blood being collected and corresponding to a specific site. For example, the team member drawing from the right IPS will wear a blue sticker and inject blood into blue-stickered vials labeled -5 $\mathrm{min}, 0 \mathrm{~min},+2 \mathrm{~min}$, and so forth. The effect of different venous flow rates on ACTH levels is compensated for by normalizing to prolactin levels in each sample. ${ }^{10,11}$ After the collection of blood, the location of each catheter is confirmed. Each catheter is then removed, followed by removal of the guide catheters. Femoral vein sheaths are removed, and manual compression is applied for 5 minutes on each side. In the setting where arteriography was required, a percutaneous closure device was deployed to secure the arterial access site before removing the venous sheath.

The ratio of central to peripheral ACTH and prolactin levels is then calculated for each time point. Central/peripheral ACTH ratios $>2$ (pre-CRH) and $>3$ (post-CRH) are used for determining a central source of $\mathrm{ACTH}^{4}{ }^{4}$ The ratio of greatest central to peripheral ACTH is then divided by the ratio of greatest central to peripheral prolactin to achieve the normalized result. ${ }^{14} \mathrm{~A}$ central/peripheral prolactin normalized ACTH ratio $>1.3$ is used as an additional indicator for a central source of ACTH. ${ }^{10,11} \mathrm{~A}$ normalized ratio $>1.4$ between left and right is used as an indicator for laterality. ${ }^{2}$

\section{Results}

The results of IPSS in 7 patients and the resulting laboratory test values along with predicted lateralization are shown in Table 1. A total of 7 patients underwent this specified protocol, of whom 5 were female. Three patients presented with a small or questionable pituitary microadenoma on MRI, and 4 others had no tumor revealed by imaging. IPSS confirmed Cushing's syndrome and was consistent with the laterality of the microadenoma in all patients in whom laterality was predicted. All patients who underwent surgery had ACTH-producing adenomas on the basis of histopathology and underwent hormonal cure. 
Patient 7 was determined not to have Cushing's disease and therefore did not undergo surgery. The cause of her Cushing's syndrome remains undetermined. Of note, patient 4 initially underwent surgery to resect a 5-mm left-sided T2-hyperintense lesion presumed to be an ACTH-secreting microadenoma but was found to have a second lesion postoperatively that was consistent with IPSS lateralization. The lateralization data gained from IPSS served to provide further evidence to support the candidacy of the small T2 hyperintensity as a second adenoma, despite the rarity of such a situation.

The upper limit of detection for ACTH at our institution is 1,250 $\mathrm{pg} / \mathrm{mL}$, making detection of the true peak ACTH above this level difficult, especially if the patient has an ACTH level $>1,250 \mathrm{pg} / \mathrm{mL}$ before $\mathrm{CRH}$ injection. In these cases, trends in raw ACTH levels bilaterally were used to determine laterality because normalizing with prolactin levels yielded misleading results in these patients, given the higher upper limit of detection for prolactin $(2,080 \mathrm{ng} / \mathrm{mL})$. This was relevant in patients 1 and 3 , as depicted in Table 1. Accurate lateralization of the tumor was achieved in all patients with ACTH levels above the upper detection limit unilaterally. In patient 5 , in whom the tumor did not lateralize, ACTH levels superseded the upper limit of detection after $\mathrm{CRH}$ administration bilaterally. This patient was found to have a left-sided tumor on surgical exploration, and although IPSS did not show an intersinus gradient $>1.4$, it was weighted toward the left side with an intersinus ratio of 1.25 in favor of the left.

\section{Illustrative Case 1}

A 29-year-old male presented with medication-resistant hypertension of 2 years' duration. He was also experiencing persistent hypokalemia, weight gain, and bruising. Work-up later revealed Cushing's syndrome. His sellar MRI showed a hypoenhancing focus in the right posterior region of the pituitary gland, and he was subsequently referred for IPSS. During the procedure, an IPS could not be identified on either side on the basis of a venogram from the jugular bulb. Arterial angiography was performed to assess the primary drainage patterns of the cavernous sinus. Venous phases of the right carotid arterial angiogram revealed the right cavernous sinus draining primarily into the pterygoid plexus anteriorly, with a small IPS (Fig. 3A). With a delayed roadmapping technique, the IPSs were identified bilaterally and cannulated. Given the significant anterior drainage through the pterygoid plexus, the microcatheter was advanced into the anterior compartment of the right cavernous sinus to sample as close as possible to the site of primary drainage. A left vertebral arterial angiogram also revealed an atretic IPS draining from the left cavernous sinus into the jugular bulb and was determined to be the side of primary drainage. This could also be visualized with a venogram from the contralateral side (Fig. 3B). A microcatheter was then positioned at the junction of the internal jugular vein and the IPS on the left (Fig. 3C). Sampling based on prolactin-normalized ACTH and trends in raw ACTH confirmed lateralization to the right.

After this, the patient subsequently underwent endoscopic endonasal exploration of the right side of the pituitary gland, which revealed an ACTH-secreting pituitary microadenoma. The patient experienced no complications and achieved hormonal cure but was later briefly readmitted for hyperkalemia caused by continuation of a potassium-sparing diuretic after achieving hormonal cure. His postoperative course was otherwise normal.

\section{Illustrative Case 2}

An otherwise healthy 33-year-old female presented with a history of unexplained weight gain. After work-up, she was determined to have Cushing's syndrome, and MRI revealed a 3-mm T2 hyperintensity at the left-central aspect of the gland, just inferior to where stalk transitions to gland (Fig. 4A). IPSS was recommended due to the small size of the lesion. Venography showed that her right cavernous sinus drained primarily through a complex venous plexus that emptied into the occipital plexus; the vertebrobasilar plexus; and, to a lesser extent, the right sigmoid sinus (Fig. 4B). On the basis of this information, the microcatheter was advanced through the venous plexus into the right cavernous sinus. Had this not been possible, sampling would have been done from within the venous plexus or where the plexus met the internal jugular vein. Another microcatheter was placed in the left IPS, which was hypoplastic but could be cannulated (Fig. 4C). IPSS subsequently revealed Cushing's disease with strong lateralization to the right.
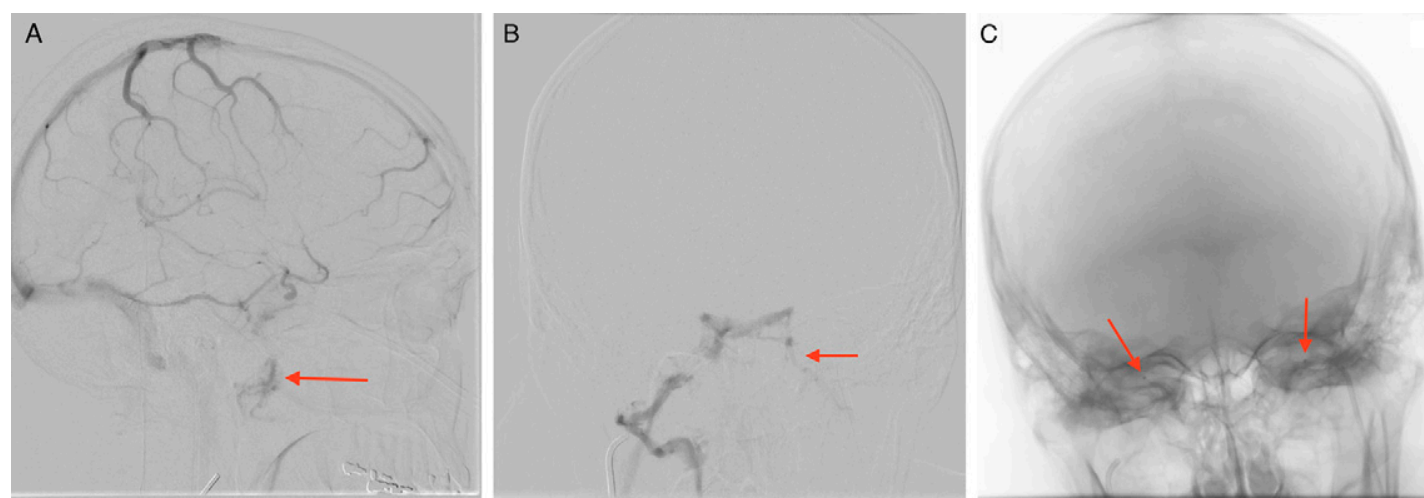

FIG. 3. A: Lateral projection of the venous phase of a right internal carotid artery digital subtraction angiogram showing anterior drainage of the right cavernous sinus through the pterygoid plexus (red arrow). B: Posteroanterior (PA) view of a venogram from the right cavernous sinus performed after identifying and cannulating the right IPS. An atretic left IPS can be visualized (red arrow). C: PA view of final catheter sampling positions in the right cavernous sinus and left IPS-internal jugular vein junction (red arrows). 

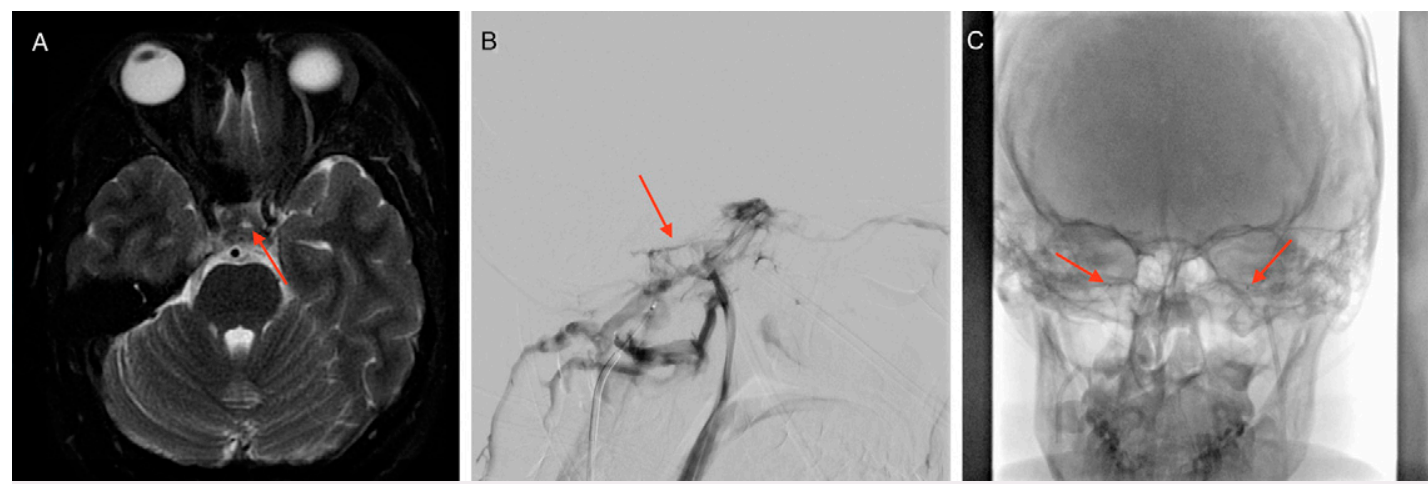

FIG. 4. A: MRI of patient 6 . Axial T2 MRI without contrast showing a small hyperintensity in a left-central location, just inferior to the infundibulum (red arrow). This hyperintensity was determined not to be a tumor intraoperatively, prompting exploration and identification of the microadenoma in the right pituitary gland. B: Lateral projection of a venogram of the right cavernous sinus showing drainage through a complex venous plexus rather than a single IPS (red arrow). C: Posteroanterior view of final catheter sampling positions showing placement in the right cavernous sinus and left IPS (red arrows).

After diagnosis, the patient underwent endoscopic transsphenoidal resection of her microadenoma. The 3-mm T2-hyperintense lesion previously seen on MRI was explored, and preliminary histological analysis of the frozen section was not consistent with an adenomatous tumor. Due to the strong lateralization toward the right determined from IPSS, the right aspect of the gland was then explored for a possible tumor. During this extended exploration, a clear focus of suspected tumor was encountered distant from the imaging-based presumed location of tumor and removed. A frozen section in this instance confirmed the findings of a pituitary microadenoma. Postoperatively, the patient had an uncomplicated course, and subsequent cortisol levels demonstrated a hormonal cure.

\section{Discussion}

\section{Observations}

Our methods differ from previous ones due to systematic site selection based on venous drainage rather than sampling from the IPS by default. Although alternate sampling sites in IPSS are known, there are no systematic approaches to selecting or identifying sites for sampling previously described. The modifications to IPSS presented here rely on knowledge of the physiology and variations in venous anatomy of the IPS. Mere presence of the IPS should not result in sampling the IPS by default. Venous drainage of the cavernous sinus can be altered by the presence of a plexiform IPS, hypoplastic IPS, or significant anterior drainage of the cavernous sinus through the pterygoid plexus. ${ }^{15,17}$ Although it has been known that cavernous sinus drainage patterns can affect or confound the results of IPS or cavernous sinus sampling, the algorithm we use here to identify drainage patterns to determine optimal sampling locations has not been described previously. ${ }^{18}$ The identification of the site of primary drainage allows targeted microcatheter positioning to increase the probability of sampling with the highest possible fidelity, thus allowing more accurate lateralization. Arteriography may also be warranted in certain cases to delineate anatomy that cannot be visualized through venography. Arteriographic enhancement of venographic data should be considered in the setting of hypoplastic petrosal sinuses because dominant antegrade drainage through the pterygoid plexus may confound negative or nonlateralizing findings. In such cases, strong consideration should also be given to advancing the catheter to the anterior compartment of the cavernous sinus to account for the influence of drainage on local hormone levels. Although cavernous sinus sampling has been shown in some studies to be equally as reliable as or less reliable than IPSS in determining lateralization, these studies did not account for variations in venous drainage before selecting sampling sites as we have done. ${ }^{8,19} \mathrm{~A}$ recent large study of lateralization using IPSS, which found a positive predictive value of $48 \%$ for lateralization, did not select for optimal sampling sites based on the site of primary drainage and defaulted to sampling from the IPS, regardless of venous drainage patterns. ${ }^{6}$ Other authors have reported success with the concept of identifying the dominant drainage pattern in a single patient, leading to successful lateralization based on external jugular vein sampling. ${ }^{15}$

\section{Lessons}

The systematic approach to identifying cavernous sinus drainage patterns and technical nuances presented here represent an improvement over previously described approaches to IPSS. Variations in venous drainage are known to result in technical failure of IPSS lateralization, and our approach aims to address this problem. ${ }^{9}$ By sampling from the sites of primary drainage rather than a predetermined anatomical location, the confounding effect of asymmetrical drainage on lateralization accuracy is reduced. We further reduce the effect of systematic error by standardizing our methods as outlined in the algorithm shown in Fig. 2. Although lateralization with IPSS remains controversial, we believe that, with this algorithm, it may become more accurate in patients with complex venous anatomy by accounting for these variations.

As noted in the 2 case vignettes, after the careful anatomical and physiological flow of the cavernous sinus was assessed for using the algorithm, the information gained from IPSS proved to be useful in the correct localization and the identification of additional tumor in situations where initial MRI-based exploration failed to identify the culprit lesion and achieve hormonal cure. The improved lateralization of pituitary microadenomas greatly reduces the need for blind explorations of the pituitary gland, thereby reducing the morbidity of pituitary surgery. A low threshold for using IPSS in cases of small or equivocal lesions on MRI can also minimize the exploration for the actual tumor, should the lesion seen on MRI 
prove to be false. We believe that the effective use of IPSS based on the algorithm presented here has contributed to the achievement of hormonal cure in all patients who underwent surgery. This was approached at our institution in a multidisciplinary fashion to ensure maximum benefit from surgery.

\section{Limitations}

Although the techniques presented here offer significant improvement in the ability of IPSS to determine laterality, there remain some limitations. Although this series demonstrates successful catheterization of the cavernous sinus through variant anatomy such as a venous plexus in place of the IPS, this may not always be technically feasible. Laboratory limitations with respect to the upper limits of detection of ACTH levels required additional analysis of data to correctly lateralize in some patients. In the case of patient 5, ACTH levels superseded the upper limit of detection at multiple time points, which prevented accurate lateralization. Dilutional studies may facilitate lateralization in cases of ACTH levels above the limit of detection. Although IPSS is a useful technique to diagnose Cushing's disease in patients with negative or equivocal MRI scans and in some instances help to lateralize the lesion, the diagnosis of cyclical Cushing's disease currently remains a challenge. $^{20}$

\section{References}

1. Lonser RR, Nieman L, Oldfield EH. Cushing's disease: pathobiology, diagnosis, and management. J Neurosurg. 2017;126(2): 404-417.

2. Lad SP, Patil CG, Laws ER Jr, Katznelson L. The role of inferior petrosal sinus sampling in the diagnostic localization of Cushing's disease. Neurosurg Focus. 2007;23(3):E2.

3. Pecori Giraldi F, Cavallo LM, Tortora F, et al. The role of inferior petrosal sinus sampling in ACTH-dependent Cushing's syndrome: review and joint opinion statement by members of the Italian Society for Endocrinology, Italian Society for Neurosurgery, and Italian Society for Neuroradiology. Neurosurg Focus. 2015;38(2):E5.

4. Oldfield EH, Doppman JL, Nieman LK, et al. Petrosal sinus sampling with and without corticotropin-releasing hormone for the differential diagnosis of Cushing's syndrome. $N$ Engl J Med. 1991;325(13):897-905.

5. Wind JJ, Lonser RR, Nieman LK, DeVroom HL, Chang R, Oldfield $\mathrm{EH}$. The lateralization accuracy of inferior petrosal sinus sampling in 501 patients with Cushing's disease. J Clin Endocrinol Metab. 2013;98(6):2285-2293.

6. Deipolyi A, Bailin A, Hirsch JA, Walker TG, Oklu R. Bilateral inferior petrosal sinus sampling: experience in 327 patients. J Neurointerv Surg. 2017;9(2):196-199.

7. Harker P, Feo-Lee O, Giraldo-Grueso M, Puentes JC. Effectiveness of bilateral inferior petrosal sinuses sampling in tumor lateralization: intraoperative findings and postoperative results. J Neurol Surg $B$ Skull Base. 2017;78(6):506-511.

8. Lefournier V, Martinie M, Vasdev A, et al. Accuracy of bilateral inferior petrosal or cavernous sinuses sampling in predicting the lateralization of Cushing's disease pituitary microadenoma: influence of catheter position and anatomy of venous drainage. J Clin Endocrinol Metab. 2003;88(1):196-203.

9. Doppman JL, Chang R, Oldfield EH, Chrousos G, Stratakis CA, Nieman LK. The hypoplastic inferior petrosal sinus: a potential source of false-negative results in petrosal sampling for Cushing's disease. J Clin Endocrinol Metab. 1999;84(2):533-540.
10. Sharma ST, Raff H, Nieman LK. Prolactin as a marker of successful catheterization during IPSS in patients with ACTH-dependent Cushing's syndrome. J Clin Endocrinol Metab. 2011;96(12):3687-3694.

11. Findling JW, Kehoe ME, Raff $\mathrm{H}$. Identification of patients with Cushing's disease with negative pituitary adrenocorticotropin gradients during inferior petrosal sinus sampling: prolactin as an index of pituitary venous effluent. J Clin Endocrinol Metab. 2004;89(12): 6005-6009.

12. De Sousa SMC, McCormack AI, McGrath S, Torpy DJ. Prolactin correction for adequacy of petrosal sinus cannulation may diminish diagnostic accuracy in Cushing's disease. Clin Endocrinol (Oxf). 2017;87(5):515-522.

13. Grant $P$, Dworakowska D, Carroll P. Maximizing the accuracy of Inferior petrosal sinus sampling: validation of the use of prolactin as a marker of pituitary venous effluent in the diagnosis of Cushing's disease. Clin Endocrinol (Oxf). 2012;76(4):555-559.

14. Mulligan GB, Faiman C, Gupta M, et al. Prolactin measurement during inferior petrosal sinus sampling improves the localization of pituitary adenomas in Cushing's disease. Clin Endocrinol (Oxf). 2012;77(2):268-274.

15. Peterson KA, Burnette CD, Fargen KM, et al. External jugular venous sampling for Cushing's disease in a patient with hypoplastic inferior petrosal sinuses. J Neurosurg. Published online January 17 , 2020. doi: 10.3171/2019.11.jns192374.

16. Graham KE, Samuels MH, Nesbit GM, et al. Cavernous sinus sampling is highly accurate in distinguishing Cushing's disease from the ectopic adrenocorticotropin syndrome and in predicting intrapituitary tumor location. J Clin Endocrinol Metab. 1999;84(5):1602-1610.

17. Miller DL, Doppman JL, Chang R. Anatomy of the junction of the inferior petrosal sinus and the internal jugular vein. AJNR Am J Neuroradiol. 1993;14(5):1075-1083.

18. Hayashi N, Kurimoto M, Kubo M, et al. The impact of cavernous sinus drainage pattern on the results of venous sampling in patients with suspected Cushing syndrome. AJNR Am J Neuroradiol. 2008;29(1):69-72.

19. Mamelak AN, Dowd CF, Tyrrell JB, McDonald JF, Wilson CB. Venous angiography is needed to interpret inferior petrosal sinus and cavernous sinus sampling data for lateralizing adrenocorticotropin-secreting adenomas. J Clin Endocrinol Metab. 1996;81(2):475-481.

20. Bonert V, Bose N, Carmichael JD. Cyclic Cushing's disease with misleading inferior petrosal sinus sampling results during a trough phase. Neurosurg Focus. 2015;38(2):E7.

\section{Disclosures}

The authors report no conflict of interest concerning the materials or methods used in this study or the findings specified in this paper.

\section{Author Contributions}

Conception and design: Prestigiacomo, Body. Acquisition of data: Matur, Smith, Lester. Analysis and interpretation of data: Prestigiacomo, Matur, Johnson, Forbes. Drafting the article: Prestigiacomo, Matur, Body, Johnson. Critically revising the article: Prestigiacomo, Matur, Body, Johnson, Smith, Bhabhra, Grossman, Forbes. Reviewed submitted version of manuscript: Prestigiacomo, Matur, Body, Johnson, Smith, Lester, Stahl, Grossman, Shirani, Forbes. Approved the final version of the manuscript on behalf of all authors: Prestigiacomo. Administrative/technical/material support: Prestigiacomo, Stahl. Study supervision: Prestigiacomo.

\section{Correspondence}

Charles J. Prestigiacomo: University of Cincinnati College of Medicine, Cincinnati, OH. presticj@ucmail.uc.edu. 